\title{
SMF: A User-Friendly Software Engine for Space-Mapping-Based Engineering Design Optimization
}

\author{
Slawomir Koziel*, Senior Member, IEEE, and John W. Bandler, Life Fellow, IEEE
}

\begin{abstract}
Although space mapping is a powerful optimization and modeling methodology, it is not always straightforward to implement, especially if one wants to use some advanced techniques and employ commercial simulators in the automatic optimization loop. The SMF system is a user-friendly space mapping software that is designed to make space mapping accessible to engineers inexperienced in this technology. SMF allows automatic or interactive space-mapping-based constrained optimization, modeling and statistical analysis. It incorporates most of the existing space mapping techniques including input, output, implicit, and frequency space mapping as well as the latest developments such as space-mapping based interpolation and advanced modeling techniques. SMF provides sockets to popular EM/circuit simulators including Sonnet em, MEFiSTo, ADS, and FEKO. In this paper we give a brief introduction to space mapping methodology as well as an exposition of the SMF system with special focus on its optimization interfaces. An SMF application example is given.
\end{abstract}

Index Terms - space mapping, space mapping software, SMF system, engineering optimization, microwave design.

\section{INTRODUCTION}

Space mapping (SM) [1]-[4] is a methodology that allows efficient optimization of expensive or "fine" models by means of the iterative optimization and updating of so-called "coarse" models which are less accurate but cheaper to evaluate. Provided that the misalignment between the fine and coarse models is not significant, SM-based algorithms typically provide satisfactory results after only a few evaluations of the fine model.

Space mapping is widely used in the optimization of microwave devices [1], [2], [4]-[7], where fine models are often based on full-wave electromagnetic simulations, whereas coarse models may be physically-based circuit models. A number of papers cover different aspects of space mapping, including the development of new algorithms ([1][4]), SM-based modeling [8]-[11], theoretical foundations ([4], [5]), etc.

In this paper we give a brief introduction to space mapping as well as an exposition of the SMF system: a user-friendly

This work was supported in part by the Natural Sciences and Engineering Research Council of Canada under Grant OGP0007239 and Grant STPGP336760, and by Bandler Corporation.

S. Koziel is with the Simulation Optimization Systems Research Laboratory, Department of Electrical and Computer Engineering, McMaster University, Hamilton, ON, Canada L8S 4K1 (e-mail: koziels@mcmaster.ca)

J.W. Bandler is with the Simulation Optimization Systems Research Laboratory, Department of Electrical and Computer Engineering, McMaster University, Hamilton, ON, Canada L8S 4K1, and also with Bandler Corporation, P.O. Box 8083, Dundas, ON, Canada L9H 5E7 (e-mail: bandler@mcmaster.ca) space mapping software engine allowing automated and interactive space mapping optimization and modeling.

\section{SPACE MAPPING OPTIMIZATION BASICS}

Let $\boldsymbol{R}_{f}$ denote the response vector of a fine model of the device of interest, which might be the evaluation of some characteristics of the device, e.g., $\left|S_{21}\right|$, at a given set of frequencies. Our goal is to solve

$$
\boldsymbol{x}_{f}^{*}=\arg \min _{\boldsymbol{x}} U\left(\boldsymbol{R}_{f}(\boldsymbol{x})\right)
$$

where $U$ is a given objective function. We consider an optimization algorithm that generates a sequence of points $\boldsymbol{x}^{(i)}$, $i=0,1,2, \ldots$, and a family of surrogate models $\boldsymbol{R}_{s}^{(i)}$, so that

$$
\boldsymbol{x}^{(i+1)}=\arg \min _{\boldsymbol{x}} U\left(\boldsymbol{R}_{s}^{(i)}(\boldsymbol{x})\right)
$$

Let $\boldsymbol{R}_{c}$ denote the response vector of the coarse model that describes the same object as the fine model: less accurate but much faster to evaluate. SM assumes that the family of surrogate models is constructed from the coarse model in such a way that the misalignment between $\boldsymbol{R}_{s}^{(i)}$ and the fine model is minimized. Let $\overline{\boldsymbol{R}}_{s}$ be a generic SM surrogate model, i.e., the coarse model composed with suitable SM transformations. At iteration $i$ the surrogate model $\boldsymbol{R}_{s}^{(i)}$ is defined as

$$
\boldsymbol{R}_{s}^{(i)}(\boldsymbol{x})=\overline{\boldsymbol{R}}_{s}\left(\boldsymbol{x}, \boldsymbol{p}^{(i)}\right)
$$

where

$$
\boldsymbol{p}^{(i)}=\arg \min _{\boldsymbol{p}} \sum_{k=0}^{i} w_{i, k}\left\|\boldsymbol{R}_{f}\left(\boldsymbol{x}^{(k)}\right)-\overline{\boldsymbol{R}}_{s}\left(\boldsymbol{x}^{(k)}, \boldsymbol{p}\right)\right\|
$$

is a vector of model parameters and $w_{i, k}$ are weighting factors. A variety of SM surrogate models is available [1]-[4], e.g., the input SM [1], in which the generic SM surrogate model takes the form $\overline{\boldsymbol{R}}_{s}(\boldsymbol{x}, \boldsymbol{p})=\overline{\boldsymbol{R}}_{s}(\boldsymbol{x}, \boldsymbol{B}, \boldsymbol{c})=\boldsymbol{R}_{c}(\boldsymbol{B} \cdot \boldsymbol{x}+\boldsymbol{c})$. Typically, the starting point $\boldsymbol{x}^{(0)}$ of the SM optimization algorithm is a coarse model optimal solution, i.e., $\boldsymbol{x}^{(0)}=\arg \min \left\{\boldsymbol{x}: U\left(\boldsymbol{R}_{c}(\boldsymbol{x})\right)\right\}$.

The space mapping optimization algorithm flow can be described as follows:

Step 1 Set $i=0$;

Step 2 Evaluate the fine model to find $\boldsymbol{R}_{f}\left(\boldsymbol{x}^{(i)}\right)$;

Step 3 Obtain the surrogate model $\boldsymbol{R}_{s}^{(i)}$ using (3) and (4);

Step 4 Given $\boldsymbol{x}^{(i)}$ and $\boldsymbol{R}_{s}^{(i)}$, obtain $\boldsymbol{x}^{(i+1)}$ using (2);

Step 5 If the termination condition is not satisfied go to Step 2; else terminate the algorithm;

Usually, the algorithm is terminated in the case of obtaining convergence or exceeding the user-defined maximum number of iterations.

\section{SMF-USER-FrIENDLY SPACE MAPPING SOFTWARE}

In order to make space mapping accessible to engineers not experienced in this technology, we have created a 
prototype user-oriented software package. SMF [12] is a GUI based Matlab system that can do space-mapping-based constrained optimization, modeling and statistical analysis. It implements existing SM approaches, including input, output, implicit and frequency SM (in particular a generalized implicit SM framework [4]). It contains drivers for simulators (Sonnet em, MEFiSTo, ADS, FEKO) that allow linking the fine/coarse model to the algorithm and make the optimization process fully automatic.

In this paper, we only focus on one aspect of SMF-a module for automatic space mapping optimization. We demonstrate the software using an example design problem.

Fig. 1 shows a block diagram of the space mapping optimization module in SMF. Optimization is performed in several steps. First, the user enters the problem arguments, including starting point, frequency sweep, optimization type and specifications. Next, the user sets up space mapping itself, i.e., the kind of space mapping to be used (e.g., input, output, implicit), specifies the termination condition, parameter extraction options, and optional constraints.

The next step is to link the fine and coarse models to SMF by setting up the data that will be used to create model drivers. Using user-provided data (e.g., simulator input files and design variable identification data), SMF creates drivers that are later used to evaluate fine/coarse models for any necessary design variable values. Model evaluation is accomplished by generating the simulator files corresponding to the required design, running the simulator and acquiring the results.

Parameter extraction, surrogate model optimization and optional trust region specific options are set in the next step using auxiliary interfaces.

Having done the setup, the user runs the execution interface, which allows the running of the space mapping optimization algorithm and visualizing the results, including model responses, specification error plots as well as convergence plots.

We demonstrate SMF using the microstrip band-pass filter [13] shown in Fig. 2. The design parameters are $\boldsymbol{x}=\left[\begin{array}{lllll}L_{1} & L_{2} & L_{3} & L_{4} & g\end{array}\right]^{T}$. The fine model is simulated in FEKO [14], the coarse model is the circuit model implemented in Agilent ADS [15] (Fig. 3). The design specifications are

$\left|S_{21}\right| \leq-20 \mathrm{~dB}$ for $4.5 \mathrm{GHz} \leq \omega \leq 4.7 \mathrm{GHz}$

$\left|S_{21}\right| \geq-3 \mathrm{~dB} \quad$ for $\quad 4.9 \mathrm{GHz} \leq \omega \leq 5.1 \mathrm{GHz}$

$\left|S_{21}\right| \leq-20 \mathrm{~dB}$ for $5.3 \mathrm{GHz} \leq \omega \leq 5.5 \mathrm{GHz}$

The initial design is the coarse model optimal solution $\boldsymbol{x}^{(0)}=\left[\begin{array}{lllll}6.784 & 4.890 & 6.256 & 5.28 & 0.0956\end{array}\right]^{T} \mathrm{~mm}$. For this problem we want to use input and output space mapping. In particular, the space mapping surrogate model is defined as $\boldsymbol{R}_{s}^{(i)}(\boldsymbol{x})=\boldsymbol{R}_{c}\left(\boldsymbol{x}+\boldsymbol{c}^{(i)}\right)+\boldsymbol{d}^{(i)}$, where vector $\boldsymbol{c}^{(i)}$ is found using parameter extraction (4), after which $\boldsymbol{d}^{(i)}$ is the residual vector evaluated by $\boldsymbol{d}^{(i)}=\boldsymbol{R}_{f}\left(\boldsymbol{x}^{(i)}\right)-\boldsymbol{R}_{c}\left(\boldsymbol{x}^{(i)}+\boldsymbol{c}^{(i)}\right)$.

We now show how to set up and run SM optimization using SMF. Fig. 4 shows the setup of the starting point, frequency sweep, optimization type and specifications within SMF. Fig. 5 shows the space mapping options interface. Here, the user can set up the termination condition for the algorithm (in terms of maximum number of iterations or fine model evaluations, or in terms of differences in the arguments or function values), space mapping to be used (checkboxes A, B, $\mathrm{C}, \mathrm{D}, \mathrm{E}$ and $\mathrm{F}$ as well as the implicit SM panel at the bottom left corner of the interface), parameter extraction options (e.g., the number of responses/Jacobians used while matching the models), as well as constraints for the coarse/fine model, regular SM parameters and implicit parameters. In our example we only use terms $\boldsymbol{c}$ and $\boldsymbol{d}$.

Fig. 6 shows the setup of the coarse and fine model drivers. As the coarse model is implemented in ADS, the user has to link to SMF the ADS netlist file corresponding to the circuit model in Fig. 3 as well as the file identifying the design variables. Linking the fine model is also simple: the user points to a FEKO input file containing the original filter design as well as to a label file containing variable labels (this information is used by SMF to identify design variables). In both cases, the user has to choose the response format. For example in the case of the FEKO model this would be the modulus of the $S_{21}$ parameter in a dB scale.

Fig. 7 shows the setup for parameter extraction, surrogate optimization and trust region options.

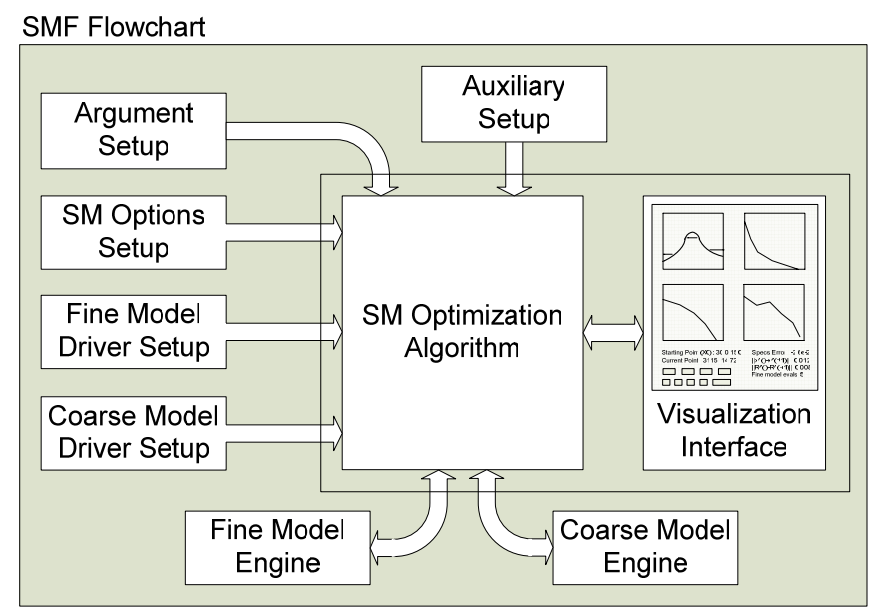

Fig. 1. Flowchart of the optimization module in the SMF system [12].

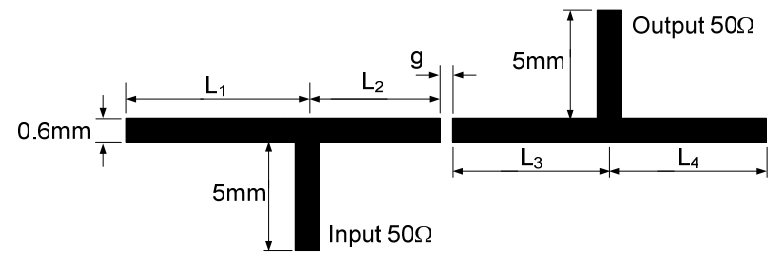

Fig. 2. Geometry of the microstrip band-pass filter [13].

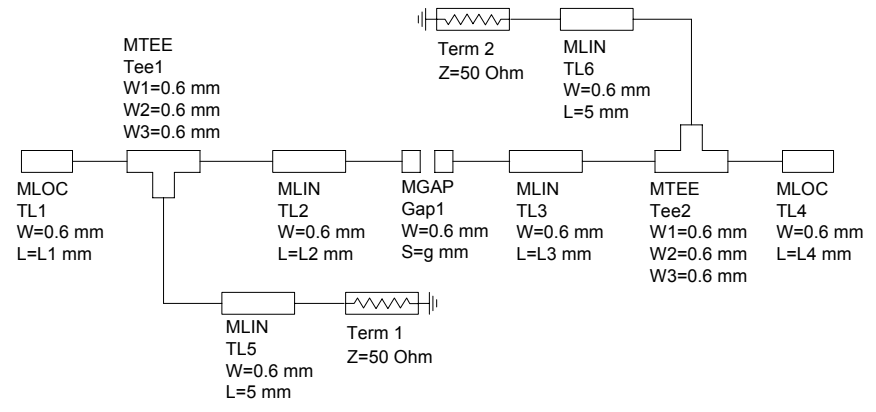

Fig. 3. Coarse model of the microstrip band-pass filter (Agilent ADS). 


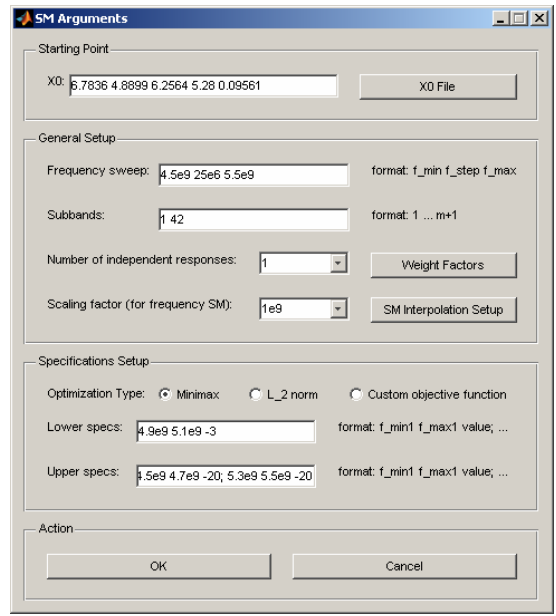

Fig. 4. Problem arguments setup in SMF.

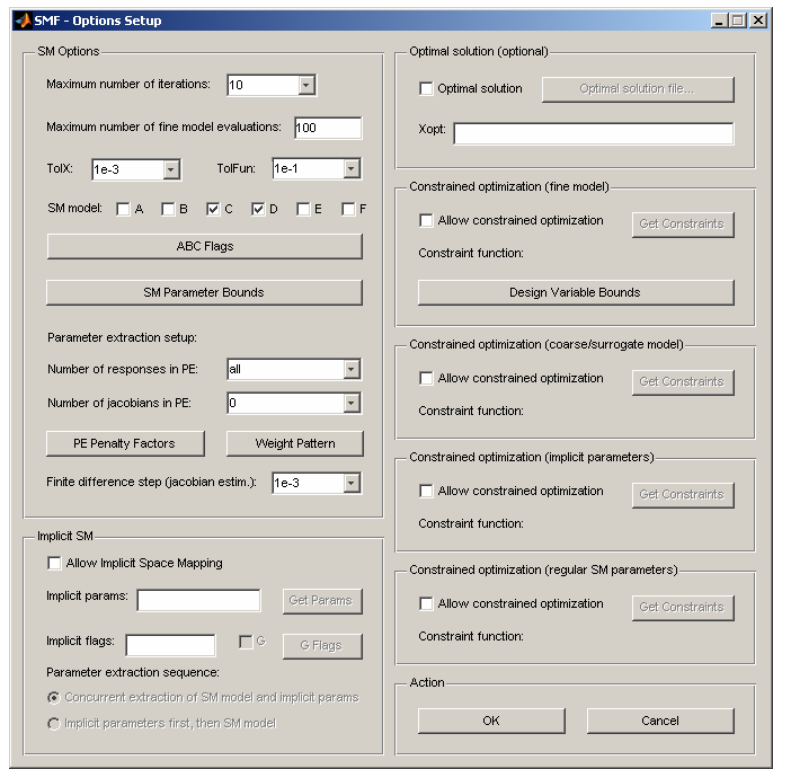

Fig. 5. Space mapping options setup in SMF.

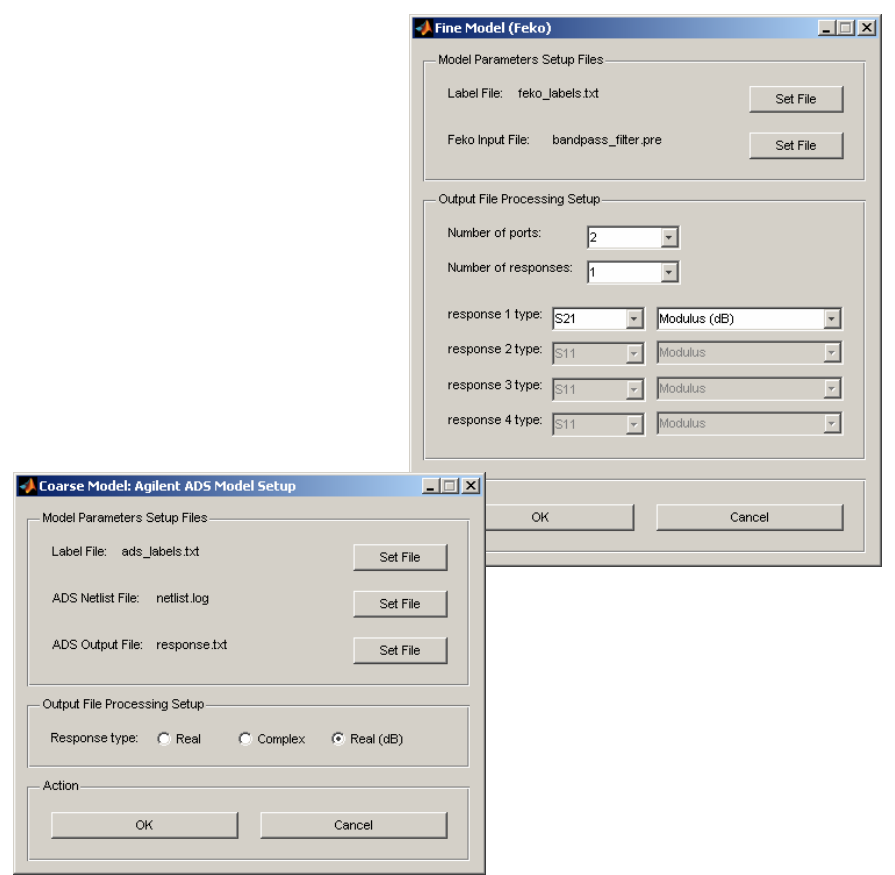

Fig. 6. ADS (left) and FEKO (right) driver setup in SMF.

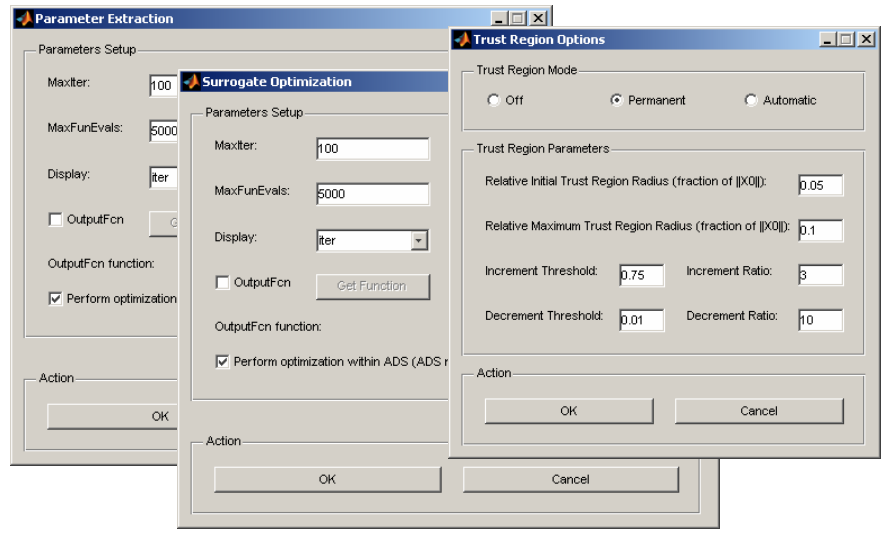

Fig. 7. Auxiliary setup in SMF (parameter extraction, surrogate optimization and trust region options).

It should be noted that SMF allows us to perform insideADS parameter extraction and surrogate model optimization [16], so that both processes can be performed using the builtin ADS optimization capability, which results in considerable savings of computational time [16].

The fine model response at $\boldsymbol{x}^{(0)}$ as well as the response at the solution obtained using SMF after 5 iterations $\left(x^{(5)}=[6.432\right.$ $\left.\left.\begin{array}{llll}4.77 & 6.176 & 4.880 & 0.0635\end{array}\right]^{T} \mathrm{~mm}\right)$ are shown in Fig. 8. The response at $\boldsymbol{x}^{(5)}$ satisfies the design specifications. The plot of specification error versus iteration number is shown in Fig. 9.

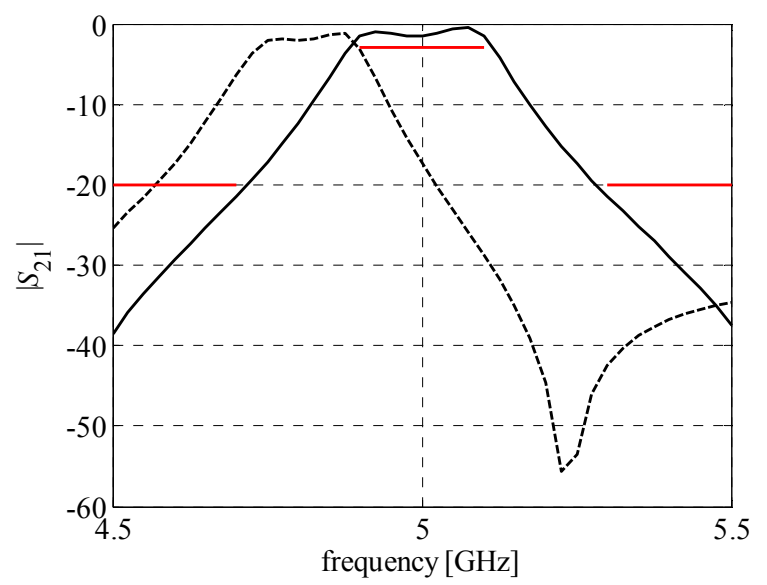

Fig. 8. Initial (dashed line) and optimized (solid line) $\left|S_{21}\right|$ versus frequency for the microstrip band-pass filter.

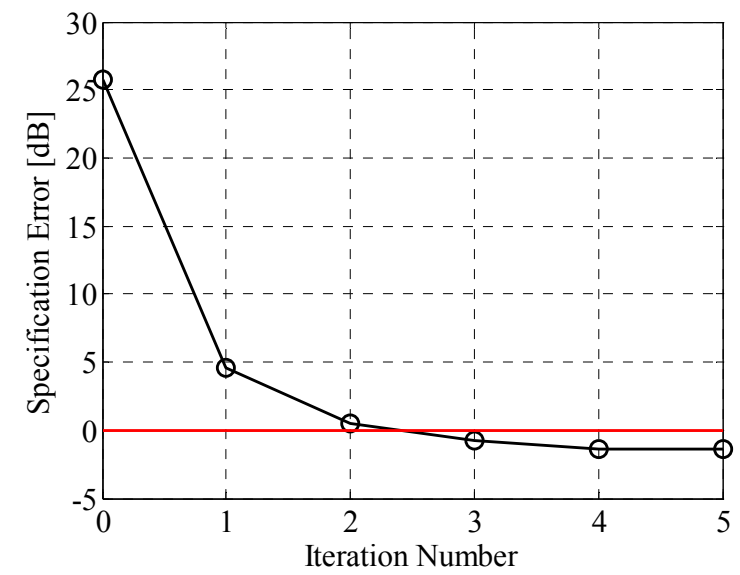

Fig. 9. Specification error vs. SM iteration number for the microstrip band-pass filter. 


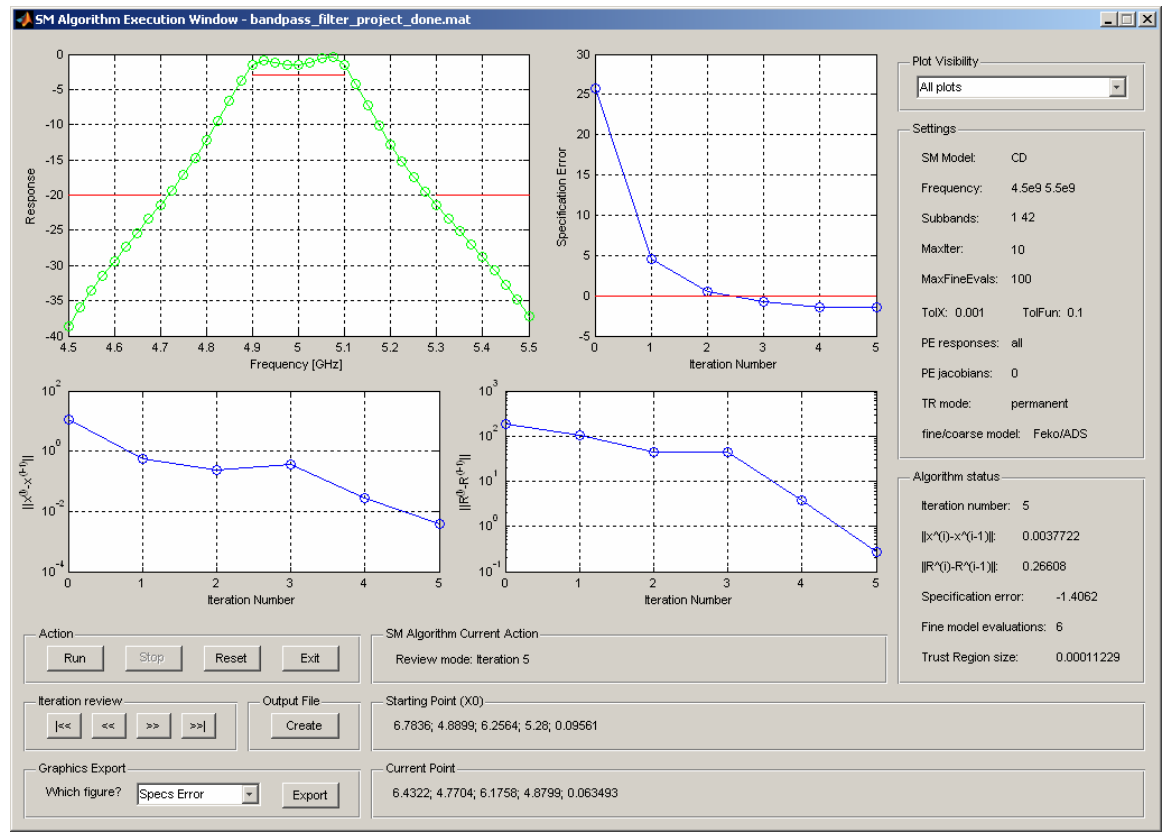

Fig. 10. Execution interface of SMF. The picture shows the interface after the optimization procedure has been accomplished.

Fig. 10 shows the execution interface of SMF, which was used to obtain these results. The plots in the interface correspond to the algorithm status after the last iteration. The top left plot shows the fine model response and the design specifications; the top right plot shows the specification error vs. iteration number; the bottom left and bottom right plots are convergence plots that show $\left\|\boldsymbol{x}^{(i+1)}-\boldsymbol{x}^{(i)}\right\|$ and $\left\|\boldsymbol{R}_{f}^{(i+1)}-\boldsymbol{R}_{f}{ }^{(i)}\right\|$ vs. iteration number, respectively. The interface contains a number of controls including buttons to run/stop optimization, export graphs, create output files as well as browse through the previous iterations of the algorithm.

Besides the presented automatic optimization module, the SMF system contains a number of other useful interfaces that allow the user to perform interactive SM optimization, SMbased modeling, frequency scaling of the coarse model, acquire fine/coarse model data, etc. Unfortunately, they cannot be described here due to the space limit.

\section{CONCLUSION}

The basics of space mapping optimization methodology are presented. The SMF system, a novel user-oriented software package that implements our SM optimization algorithms [12] is demonstrated. It provides sockets to popular commercial simulators (e.g., Sonnet em, Agilent ADS, FEKO) that allow automatic fine/coarse model data acquisition and, consequently, fully automatic SM optimization and modeling.

\section{REFERENCES}

[1] J.W. Bandler, Q.S. Cheng, S.A. Dakroury, A.S. Mohamed, M.H. Bakr, K. Madsen, and J. Søndergaard, "Space mapping: the state of the art," IEEE Trans. Microwave Theory Tech., vol. 52, no. 1, pp. 337-361, Jan. 2004.

[2] D. Echeverria and P.W. Hemker, "Space mapping and defect correction," CMAM The International Mathematical Journal Computational Methods in Applied Mathematics, vol. 5, no. 2, pp. 107-136, 2005.

[3] S. Koziel, J.W. Bandler, and K. Madsen, "Towards a rigorous formulation of the space mapping technique for engineering design," Proc. Int. Symp. Circuits, Syst., ISCAS, Kobe, Japan, pp. 5605-5608, May 2005.
[4] S. Koziel, J.W. Bandler, and K. Madsen, "A space mapping framework for engineering optimization: theory and implementation," IEEE Trans. Microwave Theory Tech., vol. 54, no. 10, pp. 3721-3730, Oct. 2006.

[5] K. Madsen and J. Søndergaard, "Convergence of hybrid space mapping algorithms," Optimization and Engineering, vol. 5, no. 2, pp. 145-156, June 2004.

[6] M.A. Ismail, D. Smith, A. Panariello, Y. Wang, and M. Yu, "EM-based design of large-scale dielectric-resonator filters and multiplexers by space mapping," IEEE Trans. Microwave Theory Tech., vol. 52, no. 1, pp. 386392, Jan. 2004.

[7] S. Amari, C. LeDrew, and W. Menzel, "Space-mapping optimization of planar coupled-resonator microwave filters," IEEE Trans. Microwave Theory Tech., vol. 54, no. 5, pp. 2153-2159, May 2006.

[8] S. Koziel, J.W. Bandler, A.S. Mohamed, and K. Madsen, "Enhanced surrogate models for statistical design exploiting space mapping technology," IEEE MTT-S IMS Digest, Long Beach, CA, June 2005, pp. 1609-1612.

[9] S. Koziel, J.W. Bandler, and K. Madsen, "Theoretical justification of space-mapping-based modeling utilizing a data base and on-demand parameter extraction," IEEE Trans. Microwave Theory Tech., vol. 54, no. 12, pp. 4316-4322, Dec. 2006.

[10] L. Zhang, J. Xu, M.C.E. Yagoub, R. Ding, and Q.-J. Zhang, "Efficient analytical formulation and sensitivity analysis of neuro-space mapping for nonlinear microwave device modeling," IEEE Trans. Microwave Theory Tech., vol. 53, no. 9, pp. 2752-2767, Sept. 2005.

[11] J.E. Rayas-Sánchez and V. Gutiérrez-Ayala, "EM-based Monte Carlo analysis and yield prediction of microwave circuits using linear-input neural-output space mapping," IEEE Trans. Microwave Theory Tech., vol. 54, no. 12, pp. 4528-4537, Dec. 2006.

[12] SMF, ver. 0.9.1, Bandler Corporation, 2007.

[13] A. Hennings, E. Semouchkina, A. Baker, and G. Semouchkin, "Design optimization and implementation of bandpass filters with normally fed microstrip resonators loaded by high-permittivity dielectric," IEEE Trans. Microwave Theory Tech., vol. 54, no. 3, pp. 1253-1261, Mar. 2006.

[14] FEKO $^{\circledR}$ User's Manual, Suite 4.2, June 2004, EM Software \& SystemsS.A. (Pty) Ltd, 32 Techno Lane, Technopark, Stellenbosch, 7600, South Africa, http://www.feko.info.

[15] Agilent ADS, Version 2003C, Agilent Technologies, 1400 Fountaingrove Parkway, Santa Rosa, CA 95403-1799, 2003.

[16] S. Koziel, Q.S. Cheng, and J.W. Bandler, "Improving efficiency of space mapping optimization of microwave structures and devices," IEEE MTT-S Int. Microwave Symp. Dig, Honolulu, Hawaii, June 2007. 